\title{
Intestinal Microbiota, the Advantages and Disadvantages of Different Methods Established for Assessing the Resident Bacterial Populations
}

\author{
Maria-Cătălina MATEI ${ }^{*}$, Victoria BUZA ${ }^{1}$, Laura Cristina ŞTEFĂNUȚ ${ }^{1}$ \\ ${ }^{1}$ Faculty of Veterinary Medicine, University of Agricultural Sciences and Veterinary Medicine, Cluj-Napoca \\ *corresponding author: catalina.matei@usamvcluj.ro
}

Bulletin UASVM Veterinary Medicine 77(1)/2020

Print ISSN 1843-5270; Electronic ISSN 1843-5378

doi:10.15835/buasvmcn-vm: 2019.0029

\begin{abstract}
At the level of the gastrointestinal (GI) tract exists a consortium of living microorganisms like bacteria, fungi, protozoa and viruses, which represent the intestinal microbiota. This term, microbiota, comes from Greek, where 'bios' means 'life' and nowadays this is the right word to use, even if in the older literature the authors used the term microflora with the same meaning (Suchodolski, 2

016). In order to describe the intestinal microbiota, there are more methods available, each of them having advantages and disadvantages. The aim of this literature study was to compare the data available regarding each method used for assessing the intestinal microbiota. Among the five methods available in the present to assess the intestinal microbiota, none of them is considered a gold standard. There are available cultural and non-cultural methods, each of them having the purpose to describe the intestinal resident bacterial populations. The most commonly used methods for characterization of the intestinal microbiota are represented by FISH- Fluorescence in situ hybridization, qPCR- quantitative real-time PCR, NGS- next-generation sequencing (e.g. 454- pyrosequencing, Illumine) and Metagenomics (shotgun sequencing of genomic DNA). In this review we made a comparison between the methods available for assessing the intestinal microbiota, showing that in the present there is not a golden standard for this and that the methods used have advantages and disadvantages.
\end{abstract}

Keywords: bacterial culture, microbiota, molecular biology

\section{Introduction}

Resident microorganisms are abundant in mammals' hosts, the number of microbial cells being 10 times higher than the total number of host cells (Gibson and Roberfroid, 1995).

The gastro-intestinal (GI) microbiome is defined as a complex group consisting of bacteria, arcanobacteria, fungi, protozoa and viruses. Those microorganisms colonize the gastro-intestinal tract of all mammals. Nowadays, numerous studies conducted in both human and veterinary medicine have shown that the microbiome is involved in a large number of vital and physiological processes. Some examples are represented by maintaining body homeostasis, metabolic processes, GI epithelial health, immunological defense and even neuro-behavioral development. More than that, the microbial genome improves the metabolic capacity of the host, being an important factor in the development of the physiological processes of the colonized organism (Barko et al., 2018).

The term microbiota, comes from Greek, where 'bios' means 'life' and nowadays this is the right word to use, even if in the older literature the authors used the term microflora with the same meaning (Suchodolski, 2016). In the past, the term microflora was considered synonymous with microbiota (Schmitz and Suchodolski, 2016). Joshua Lederberg defined the concept of the microbiome, defining this term as „the ecological community of 
commensals, symbionts and pathogenic microorganism that literally share the body" (Lederberg and McCray, 2001). Currently, the term microbiota is used in order to describe bacterial communities at the level of mucous membranes or other body membranes, such as skin. Microbiota and microbiome are often considered as overlapping terms. However, the term microbiome is currently used as a reference to the entire genetic mass of the microorganism and can be defined in this context as the genome of the microorganisms (Ten and Netea, 2014).

The microbiome is represented also by fungi. Compared to the available data on resident bacterial populations and their role in maintaining host homeostasis, the information about fungi is much less represented. The populations of fungi that physiologically colonize the GI tract form the micobiome (Li et al., 2017). Studies available now give us an overview about the predominant phyla. At the same time, researchers concluded that fungi are detectable in all GI segments in $70 \%$ of healthy subjects (Schulze and Sonnenborn, 2009).

In order to understand the complex relationships between microbiome and the host and how it is implicated in disease, there has been developed new methods, culture-independent techniques for quantification and for phylogenetic investigation. If there is a comprehensive knowledge of the composition and the action of the microbiome, a better understanding of the relationship between host's health/disease status and the resident microorganisms will be revealed (Fraher et al., 2012).

At the beginning of the research in the microbiome field, bacterial culture and biochemical typing were largely used and even considered the gold standard for the identification of bacterial species. In time, more exact methods were developed, being culture-independent techniques, which were able to give us a clear image about this domain (Fraher et al., 2012). The culture-independent techniques (like denaturing gradient gel electrophoresis- DGGE, fluorescence in situ hybridization- FISH, next-generation sequencing- NGS) are more specific because they are based on sequence divergences of $16 \mathrm{~S}$ rRNA- small subunit ribosomal RNA. This is why those methods are able to show the microbial diversity and, at the same time, to give a qualitative and quantitative characterization of the bacterial species. Moreover, using these new techniques, an image about the changes of microbiome in relation to disease is available (Fraher et al., 2012).

The importance of gastro-intestinal microbiome is, without question, a significative one. But, in order to understand all the implications between the resident bacterial populations and the host, it is mandatory to assess the composition of intestinal microbiota. Over time, researchers used different methods to identify and characterize the microbiome. All of these methods have improved over time, in order to give more exact results and a better understanding regarding the link between microbiome and host. However, given the variety of phyla implicated in this process and the differences between each microorganism group, there is not establish a method that can be considered a golden standard for assessing the microbiome. The aim of this literature review article was to compare the most common methods used for assessing the intestinal microbiota, and to show the advantages and disadvantages for each method.

\section{Methods used for assessing the resident bacterial populations}

\section{What sample do we use?}

In order to assess intestinal microbiota, using different techniques, first it is important to take into consideration the method of sampling. Most of the studies conducted by now on dogs used stool samples, intestinal mucosal brushing or luminal content (Suchodolski et al., 2008). It is known that the abundance and composition of bacterial phyla varies along different segments of the gut in dogs (Barko, 2018). This is why the results from stool sample and mucosal brushing or luminal content may be different, even if the same method is used. Considering that obtaining samples from mucosal brushing or even obtaining luminal content can be difficult in dogs, those methods are not suitable for a routine use, while stool samples are easy to collect. Those aspects can explain why the most studies in dogs have focused on the characterization of fecal microbiome.

Another concern regarding sampling is linked with the possibility of proliferation of certain microorganisms, thus the sample might no longer reflect in an accurate way the microbiome composition in vivo. Moreover, freezing stool sample before examination can alter the microbial 
composition, so fresh stool sample should be used when possible. If this is not possible, when using frozen stool samples, should be take into consideration that the levels of Bacteroidetes can be reduced (Fraher et al., 2012).

\section{Cultural techniques}

Starting with the early 20 th century, cultural techniques were used in order to assess the intestinal resident bacterial population. This approach was considered a gold standard until 1990s, when other non-cultural techniques were developed. The present knowledge on the field of intestinal microbiome lead us to the conclusion that results obtained using this method are not satisfying because the culture alone can give only a limited view of the diversity of the microbiome. This fact can be explained by the fact that less than $30 \%$ of the resident bacterial populations have been cultured by now. Even if, since the beginning of using this technique there were made some improvements in the phenotyping of isolated bacteria according to their fermentation ability and their requirements for in vitro growth, there is not enough information available for culturing the rest of $70 \%$ of bacteria. They are not unculturable, but maybe the conditions required for their growth have not yet been developed or understood. Another explanation can be that some microorganisms are dependent to the metabolic activity of other microorganisms for growth, this being a limitation for the pure-culture techniques (Fraher et al., 2012).

As it is known by now, the anaerobic bacteria need special cultural conditions, so in many cases this bacterial type is hard to be cultured. This is a very important fact for the veterinary medicine field, given the situation that most of the bacterial populations resident at the level of the canine and feline GI tract are anaerobic bacteria (Suchodolski, 2016).

The main advantage of this method is that it is cheap and does not require a complicated infrastructure or investment to be used. Moreover, it can be used on a daily basis, on a regular equipped laboratory. The disadvantages of culture method are represented by the intensive labor, the limited view given over the populations involved in the microbiome.

\section{Non-cultural techniques}

Since 1990s, the cultural methods started to be replaced step by step by new cultureindependent techniques which were developed in order to rich our knowledge about the microbiome. Almost all of these techniques are based on the DNA extraction and the amplification of the $16 \mathrm{~S}$ ribosomal RNA genes. This $16 \mathrm{~S}$ ribosomal subunit is a component of the $30 \mathrm{~S}$ subunit of a ribosome. Through the cytoplasm of a bacterial

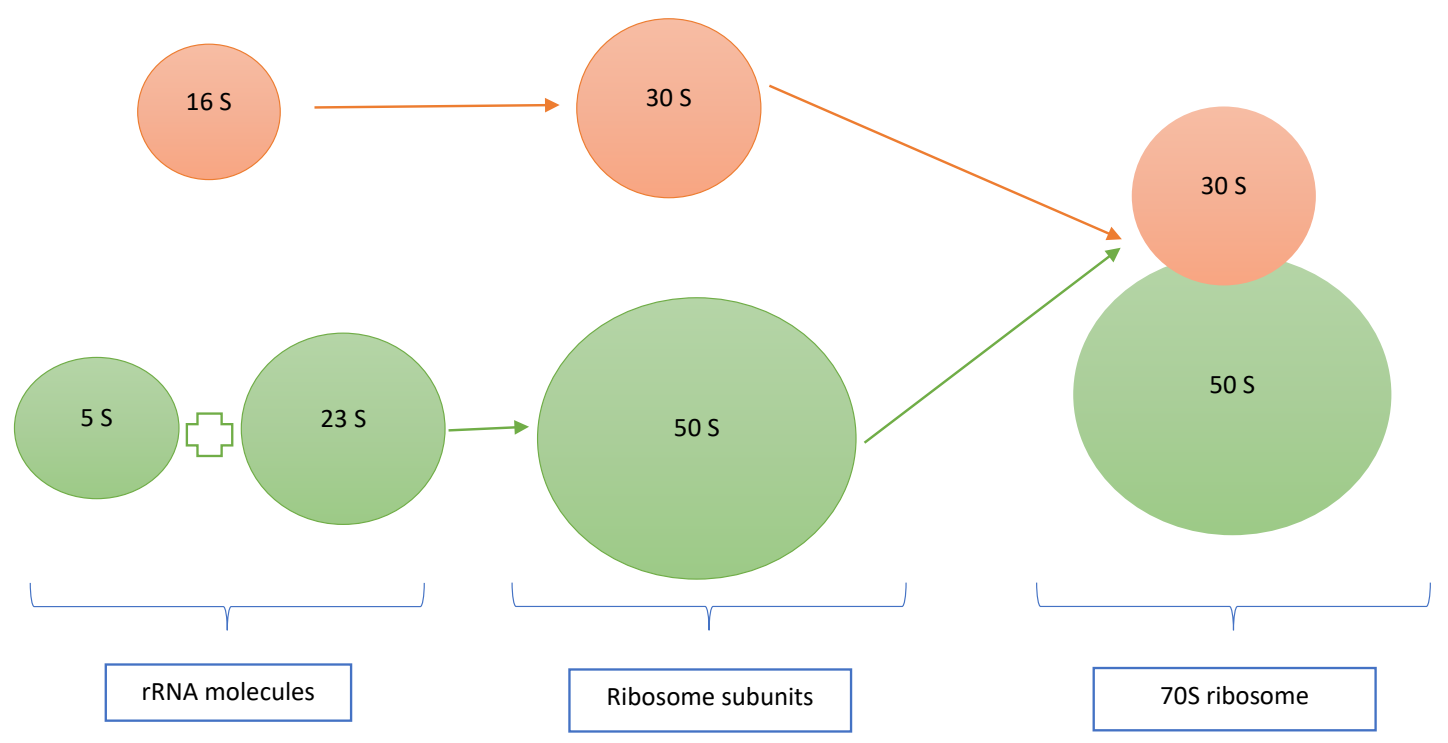

Figure 1. The 70S ribosome composition

The 16S rRNA molecule is a component of the 30S ribosome subunit; the 16S rRNA molecule is the targeted one in novel non-cultural techniques used now in order to assess the microbiome and not only 
cell are dispersed the 70S ribosomes. Those are composed of two major subunits, represented by the $50 \mathrm{~S}$ subunit (formed by two RNA molecules5S and 23S) and the 30S subunit- known as the small subunit, which contains a single molecule of rRNA16S (Figure 1). This 16s rRNA has many functions, but one of the most important is the initiation and the extension of the protein synthesis. It is known that rRNA contains different regions that vary between different bacteria, which allow us to do the phylogenetic identification, even if it is highly conserved through the bacterial species. This is why techniques that target the 16S RNA genes are useful in the bacterial identification. From all these techniques, we can identify two large categories: techniques that can phylogenetically identify the components of the intestinal microbiome and/ or techniques that can quantify them (Fraher et al., 2012). However, key information in assessing the GI microbiome is to know that some bacterial groups may have a low abundance. Pathogenic bacteria are often found in a low proportion of the total bacteria in the intestine, so, due to this aspect, they may escape identification even if the technique used has a high throughput. This is why it is recommended to be used additional use of group specific PCR primers in order to quantify or detect bacterial groups with low abundance (Suchodolski, 2016).

\section{Polymerase chain reaction}

PCR (polymerase chain reaction) was a huge revolution in the medical field. Themain mechanism of action is the amplification of $16 \mathrm{~S}$ rRNA. In case of qPCR it is involved also the quantification of $16 \mathrm{~S}$ rRNA, giving a color reaction when the fluorescent compound from the reaction mixture binds to double-stranded DNA. It is a technique that is very useful in a lot of cases. However, in the intestinal microbiome assessment, PCR alone has some limitations. If the goal is the assessment of the intestinal microbiome, quantitative PCR (qPCR) is more suitable for use. This variation of the PCR technique is able to amplify DNA and to reliably quantify the amount of DNA present in the sample, the targeted organisms being detected in realtime (Suchodolski, 2016). The whole procedure is standardized and it is composed from physical, chemical and biological steps (Fraher et al., 2012).

The advantages of the qPCR techniques in microbiome characterizations are represented by the fact that this method is fast, inexpensive, and has a turnaround of a few hours, so it is a rapid technique. Moreover, it is able to do a phylogenetic identification and, at the same time a quantification of bacterial groups in the processed sample (Suchodolski, 2016).

The disadvantages of this technique can be divided in two main categories. The first one is related to the possible bias linked to the technique. This problem can occur in any of the steps: physical, chemical or biological one. The samples can be very easily contaminated. Moreover, some Grampositive bacteria may require special conditions for the bacterial cell wall lysis because it is thicker than the Gram-negative bacteria cell wall, and this can lead to an excessive fragmentation of Gramnegative bacteria chromosomal DNA. In this case, the difference between the required conditions for the lysis can lead to a tainted image of the microbial composition. The second big disadvantage is that, in order to assess all the phyla, all the primers used must be designed to target all the phyla. Using targeted primers make it impossible to identify unknown species. At the same time, the primer design can be difficult if there are no enough information about the intestinal microbiota of the studied animal. Moreover, this technique requires an intensive labor (Fraher et al., 2012).

\section{Fluorescent in situ hybridization}

Fluorescent in situ hybridization (FISH) is a technique used in the assessment of the intestinal microbiota, but mostly in research than in clinical practice. In human medicine, it is mainly used in order to compare the GI microbiome in health and disease, with the purpose of assessing the differential abundance of the microorganism's groups (Fraher et al., 2012).

This method uses oligonucleotide probes which are fluorescent dye-labeled, those being hybridized to rRNA sequence present in the bacterial cells (Suchodolski, 2016). This technique can be performed on the stool sample directly. The main steps that are followed in order to proceed this technique are represented by sample denaturation and fixation in a hybridization solution, then the addition of the fluorescently labelled oligonucleotide probes and incubation at $67-75^{\circ} \mathrm{C}$ in a hybridization solution, typically overnight. The final step is represented by the identification of the targeted species using flow 
cytometry. From all these steps, the crucial one in order to ensure optimal results is represented by fixation, this being made usually using crosslinking agents like aldehydes or precipitating agents like ethanol, or a mixture between the two (Fraher et al., 2012).

FISH technique is able to identify and quantify the bacteria, and at the same time, to visualize the bacteria localization in tissue- luminal, cell-adherent or mucosa invasive. Because this technique is based on microscopic counts rather than DNA amplification, at the present moment, it is considered to be the most precise method that should be used in order to quantify resident GI bacterial groups (Suchodolski, 2016). FISH in combination with flow cytometry is considered a high-throughput method to assess the GI microbiome (Fraher et al., 2012).

The advantage of this method is that it is able to identify the epithelial location of the bacteria- intracellular, adherent or epithelium invasive (Suchodolski, 2016). Moreover, it is a semiquantitative method and it is not very time consuming (Fraher et al., 2012).

The disadvantages of this method are that every FISH probes must be developed for each bacterial group and, at the same time, practicing this technique requires an intense labor. Moreover, FISH technique does not allow a high throughput sample analysis (Suchodolski, 2016).

\section{Next generation sequencing}

Next generation sequencing (NGS) was launched in 2005 as the first commercial massively parallel pyrosequencing platform, being the first step on the new era of high-throughput genomic analysis (Voelkerding et al., 2009).

NGS is based more or less on the same steps as the classic DNA sequencing, until a certain point. The bacterial DNA from a sample is extracted and then amplified using universal primers. Then, the amplicons obtained are sequenced using a high throughput sequencer. This difference makes this technique able to identify, at least theoretically, all the bacterial groups which are present in the analyzed sample. This method can be considered as a semi-quantitative method. But, given the high cost and the complex infrastructure that this technique requires, it is not used for now as a current method to assess the GI microbiome, neither in veterinary medicine, nor in human medicine (Suchodolski,
2016). At this time, there are available different technologies that use the NGS mechanism of action. 454 Pyrosequencing® (Roche Diagnostics GMBH Ltd, Mannheim, Germany) and Illumina ${ }^{\circledR}$ (Illumina, San Diego, CA, USA) are the most popular. Another technology available at the present moment is represented by $\mathrm{SOLiD}^{\mathrm{TM}}$ from Life Technologies, Carlsbad, CA, USA. These technologies are applied directly to amplicon DNA or total DNA community, being able to identify all bacterial groups in the sample (Fraher et al., 2012). The main difference between those three technologies is represented by the used material. 454 Pyrosequencing ${ }^{\circledR}$ uses beads, Illumina ${ }^{\circledR}$ uses slides and SOLiD $^{\mathrm{TM}}$ uses solid surfaces.

In the case of 454 Pyrosequencing $®$, considered the most frequent used, the fragmented 16S rRNA amplicon is fixed on beads, each bead being able to attach only one DNA fragment. Then, the fragments are amplified and after a few steps, when a nucleotide is incorporated by the polymerase, pyrophosphate will be released. After that, a chemiluminescent signal will be the result after the addition of luciferase (a substance that will produce a color reaction), and this signal will be read into a pyrogram which will be analyzed and will show the order of the nucleotide bases. By comparing with the available databases, the identified order of the nucleotide bases will be categorized and the phylogenetic identification will be completed (Fraher et al., 2012). Using this technique, it is possible to sequence about 500 million bases, the accuracy being at least 99\% in a single run (Voelkerding et al., 2009). If the sequences read are shorter, many more can be read and given this situation, all the bacteria that are in a low abundance are able to be detected. If the massive parallel sequencing was already done for a genome from a species which has an available genome as a reference, the price of this technique is about 10 times low than to sequence a novel microbial genome (Fraher et al., 2012).

Among the advantages of NGS of 16S rRNA genes technique we can note the capacity to indicate relative changes in a bacterial community regarding the bacterial groups. Moreover, it is able to identify all the bacterial groups by a phylogenetic identification, even the unknown bacteria (Suchodolski, 2016, Fraher et al., 2012). Moreover, it is a fast technique and has the advantage to provide quantitative data (Fraher et al., 2012). 
The disadvantages of this technique are that it requires specialized professionals and advanced bioinformatics skills and infrastructure. More than that, it has a long turnaround and a high cost. In some cases, due to the used reagents, the obtained results may give false positive signals (Suchodolski, 2016). Moreover, if it is necessary to sequence de novo a microbial genome the costs can be high.

\section{Metagenomics}

At the present time, there are available more 'omics' approaches in order to understand the GI microbiota. So, when we speak about metagenomics, or environmental genomics or community genomics, we refer to the technique that has the purpose to study the metagenome. This is the total pool of genomes from the studied environment, in this case the animals GI tract. Another 'omics' approach is represented by metabolomics, which is the technique that studies the metabolome. This is the total amount of metabolites that are present in the studied biological sample. Then, it is used metaproteomics, known as community proteomics/ proteogenomics or environmental proteomics too, which is the technique that studies the metaproteome. This is the collective assembly of proteins which are present in a genome or expressed by a cell, tissue or the entire organism. Least but not last, now is used also metatranscriptomics, which is the study of the metatranscriptome. This is the collective set of mRNA molecules in the studied biological sample. By using all this approaches together there is a high chance to get a better understanding of the GI microbiome and its role in the host health (Fraher et al., 2012).

Metagenomics is a new technique which can be used in the characterization of the GI microbiome. It is based on fragmenting genomic DNA and then sequencing it on a random manner, using a high throughput sequencer. The fact that the DNA is sequenced without PCR amplification makes this method different from the others non-cultural techniques. Moreover, this snapshot of the gene pool from a bacterial community provides valuable information about the functional role of the microbial community. This is an important tool in order to understand the impact of the GI microbiome on the host's health (Suchodolski, 2016). Moreover, using metagenomics the scientists are able to understand better the microbiome differences between a healthy and a diseased host.
The beginning of metagenomics technique dates in 1998 when it was first used in the study of soil microbiota by the researcher named Handelsman (Handelsman et al., 1998). After her approach, this technique started to gain attention and it was used in important research projects regarding the microbiome, including the wellknown Human Microbiome Project, funded by the National Institutes of Health in the United States (Peterson et al., 2009). What makes this technique unique is the main mechanism of action represented by the sequencing of a representative sample of all DNA fragments in the sample, not just particular DNA fragments (Fraher et al., 2012).

The microbiome shotgun sequencing technique is now considered to be a good approach in order to assess the GI microbiome. This method involves the whole community DNA sequencing that involves random fragmentation of DNA, followed by the sequencing of the obtained DNA fragments and, at the end, the reconstruction of overlapping sequences in order to assemble those fragments into a continuous sequence. Using this technique, the result obtained is able to give data about the genetic diversity and functions of the GI microbiota, while the other techniques presented are able only to give information about genetic diversity of the microbial community. This fact has a great importance in order to understand and to make correlations between the microbiome and the health or disease status of the animal (Fraher et al., 2012).

The advantages are represented by the capacity to provide information regarding the functional genes present in sample and, at the same time, identification of the bacteria (Suchodolski, 2016). Having this information together, the understanding between the microbiome and the host health or disease status became more easily to understand (Fraher et al., 2012).

The disadvantages are mainly linked with the high costs involved and with the complex infrastructure needed to proceed this kind of tests (requires advanced bioinformatics) (Suchodolski, 2016). Moreover, when the shotgun sequencing technique is used, given the fact the large amount of data generated requires an intense computational work, it cannot be performed unless a specialized laboratory is involved (Fraher et al., 2012).

As we can see, there is not a single and ideal method to assess the microbial community that 
forms the GI microbiome. Each method used in the present has its advantages and disadvantages. Moreover, in order to avoid biases, it is very important to take into consideration the former steps, before the sample analysis, and the most important step is the sampling (Figure2).

Most of the non cultural techniques requires special equipped laboratories and research infrastructure, but are able to provide better results compared with the classic, cultural technique which is cheaper and does not have the same requirements.

\section{Conclusion}

We are on an early stage in understanding the close connection between the GI microbiome and the host's general health. This is why the characterization of the GI microbiome should be an easy approach even in research or in clinical cases.
As we could see, until now it is not defined a golden standard regarding the assessing methods of the resident bacterial communities at the level of the intestine. Every method which is used nowadays has its advantages and disadvantages, and this is why none of them can be considered the perfect one. The only way to obtain a complex image about the GI microbiota is to combine more methods in order to find out more exactly which bacterial groups are involved, which is the quantity of each group and which is their functional abilities. This solution has a lot of limitations, even in research, and this makes it almost impossible to use it in clinical practice. Moreover, all the techniques used in the present are designed to target the bacterial populations, but, as it is known, in the composition of the microbiome are included also fungi and viruses. An important aspect regarding to the GI microbiome is to take into consideration

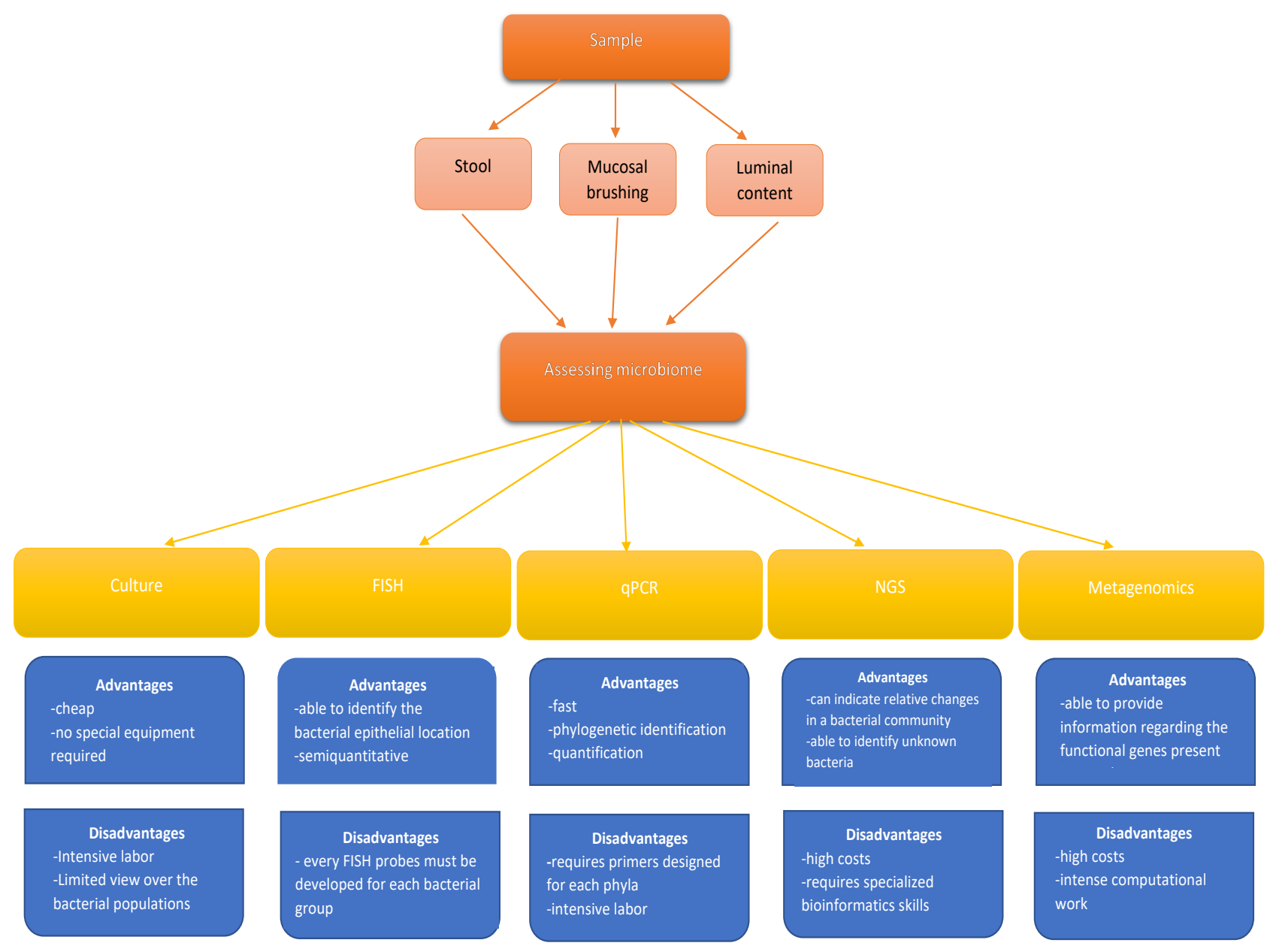

Figure 2. The advantages and disadvantages for the main methods used in the present in order to assess the GI microbiome. 
the method used to assess it when different results are compared.

Acknowledgments. This research did not receive any specific grant from funding agencies in the public, commercial, or not-for-profit sectors.

\section{References}

1. Barko PC, Michael MA, Swanson KS, Williams DA (2018). The gastrointestinal microbiome: a review, Journal of Veterinary Internal Medicine, Vol 32: 9-25.

2. Fraher MH, O’Toole PW, Eamonn M, Quigley M (2012). Techniques used to characterize the gut microbiota: a guide for the clinician, Nat. Rev. Gastroenterol. Hepatol. 9: 312-322;

3. Gibson GR, Roberfroid MB (1995). Dietary modulation of the human colonic microbiota: introducing the concept of prebiotics, Journal of Nutrition, 125: 1401- 1412

4. Handelsman, J, Rondon MR, Brady SF, Clardy J, Goodman RM (1998). Molecular biological access to the chemistry of unknown soil microbes: a new frontier for natural products. Chem. Biol. 5: 245-249.

5. Lederberg J, Mccray A (2001). 'Ome Sweet 'Omics - A Genealogical Treasury of Words, Scientist Inc.: Philadelphia, PA, 15: 8.

6. Li J, Daiwen C, Bing Y, Jun H, Ping Z, Xiangbing M, Jie Y, Junqiu L, Gang T, Zhiqing H, Yuheng L (2017). Fungi in Gastrointestinal Tracts of Human and Mice: from Community to Functions, Microb Ecol, https://doi. org/10.1007/s00248-017-1105-9;

7. Peterson J, Garges S, Giovanni M, Mcinnes P, Wang L, Schloss JA, Bonazzi V, Mcewen JE, Wetterstrand KA (2009). The NIH Human Microbiome Project, Genome Res. 19: 2317-2323.

8. Schmitz S, Suchodolski J (2016). Understanding the canine intestinal microbiota and its modification bt pro, pre- and synbiotics- what is the evidence?, Veterinary Medicine and Scienc, 2: 71- 94.

9. Schulze J, Sonnenborn U (2009). Yeasts in the gut: from commensals to infectious agents. Dtsch Arztebl Int 106:837-842

10. Suchodolski JS, Camacho J, Steiner JM (2008). Analysis of bacterial diversity in the canine duodenum, jejunum, ileum, and colon by comparative 16S rRNA gene analysis. FEMS Microbiology Ecology 66: 567-578.

11. Suchodolski JS (2016). Diagnosis and interpretation of intestinal dysbiosis in dogs and cats, The Veterinary Journal 215: 30- 37

12. Ten OJ, Netea MG (2014). The bacteriome-mycobiome interaction and antifungal host defense, European Journal of Immunology 27: 3182-3191.

13. Voelkerding KV, Dames SA, Durtschi JD (2009). Nextgeneration sequencing: from basic research to diagnostics, Clin. Chem. 55: 641-658. 